Pacific

Journal of

Mathematics

ON THE DENSITY THEOREM FOR THE SUBDIFFERENTIAL OF CONVEX FUNCTIONS ON HADAMARD SPACES

Mina Movahedi, Daryoush Behmardi

AND SEYEDEHSOMAYEH HoSSEINI 


\title{
ON THE DENSITY THEOREM FOR THE SUBDIFFERENTIAL OF CONVEX FUNCTIONS ON HADAMARD SPACES
}

\author{
Mina Movahedi, DARYOUSh BehmaRdi \\ AND SEYEDEHSOMAYEH HOSSEINI
}

\begin{abstract}
We introduce a dual space for any geodesically complete Hadamard space. By using this notion we give a new definition of the subdifferential of convex functions on geodesically complete Hadamard spaces. Some properties of this subdifferential, such as a density theorem, are proved.
\end{abstract}

\section{Introduction}

Nondifferentiability appears naturally in different areas of mathematics and arises explicitly in the description of various modern technological systems. Nonsmooth analysis studies the local behavior of nondifferentiable functions and sets lacking smooth boundaries. Generalized gradients or subdifferentials refer to several setvalued replacements for the usual derivative which are used in developing differential calculus for nonsmooth functions.

Nondifferentiable functions are often considered on finite-dimensional or infinitedimensional Banach spaces. Here, the linear structure plays a central role. Attempts have been made to replace Banach spaces with Riemannian manifolds and develop a subdifferential calculus; see [Hosseini and Pouryayevali 2011; 2013a; 2013b; 2013c]. Shafrir [1992] gave a definition of the coaccretive subdifferential of a convex function defined on a Hilbert ball. His approach involves the structure of $(B, \rho)$ as a Hilbert manifold, where $\rho$ is the hyperbolic metric on $B$; see also [Kopecká and Reich 2010, p. 188].

Unlike Riemannian manifolds, Hadamard spaces are not equipped with a Riemannian metric. Hence, we need new tools to construct a suitable dual space in order to define subdifferentials of functions on Hadamard spaces. B. Ahmadi Kakavandi and M. Amini [2010] defined a dual space for an Hadamard space using the concept of bound vectors. They defined a pseudometric $D$ on $\mathbb{R} \times \mathcal{X} \times \mathcal{X}$, where $\mathcal{X}$ is an Hadamard space, and considered the pseudometric space $(\mathbb{R} \times \mathcal{X} \times \mathcal{X}, D)$ as a subspace of the pseudometric space $(\operatorname{Lip}(\mathcal{X}, \mathbb{R}), L)$ of all real-valued Lipschitz

MSC2010: primary 53C23; secondary 47H05.

Keywords: Hadamard spaces, convex functions, subdifferential. 
functions. Then, they defined an equivalence relation on $\mathbb{R} \times \mathcal{X} \times \mathcal{X}$, where the equivalence class of $(t, a, b)$ is

$$
[t \overrightarrow{a b}]:=\{s \overrightarrow{c d}: t\langle\overrightarrow{a b}, \overrightarrow{x y}\rangle=s\langle\overrightarrow{c d}, \overrightarrow{x y}\rangle \text { for } x, y \in \mathcal{X}\}
$$

After introducing a dual metric space to $\mathcal{X}$,

$$
\mathcal{X}^{*}:=\{[t \overrightarrow{a b}]:(t, a, b) \in \mathbb{R} \times \mathcal{X} \times \mathcal{X}\},
$$

they defined a notion of the subdifferential for a proper function on an Hadamard space.

Here we present a new dual for any Hadamard space and prove a density theorem for the subdifferential of lower semicontinuous convex functions on Hadamard spaces, generalizing the classical one for Hilbert spaces [Clarke et al. 1998]. Our approach differs from the one in [Ahmadi Kakavandi and Amini 2010]: we use the notion of geodesics, defining the dual $\mathcal{X}^{*}$ as the disjoint union of the sets $\mathcal{X}_{x}^{*}$ over $x \in \mathcal{X}$, where $\mathcal{X}_{x}^{*}$ contains all unit speed geodesics of $\mathcal{X}$ starting at $x$. The subdifferential of a function $f$ at a point $x$ is defined as a subset of $\mathcal{X}_{x}^{*}$. This property is not visible in Ahmadi Kakavandi and Amini's definition of the subdifferential. This leads us to the claim that the subdifferential of convex functions defined in this paper is an analogue of the concept of the subdifferential of convex functions in Riemannian manifolds and Hilbert balls.

We assume that $\mathcal{X}$ is a geodesically complete Hadamard space with a metric $d$. Recall that a geodesic in $\mathcal{X}$ is a curve of constant speed which is locally minimizing. We say $\mathcal{X}$ has nonpositive curvature (in the sense of Alexandrov) if every point $p \in \mathcal{X}$ has a neighborhood $U$ with the following properties:

(i) For any two points $x, y \in U$ there is a geodesic $\sigma_{x}^{y}:[0,1] \rightarrow U$ from $x$ to $y$ of length $d(x, y)$.

(ii) For any triple of points $x, y, z \in U$, we have

$$
d^{2}(z, m) \leq \frac{1}{2}\left(d^{2}(z, x)+d^{2}(z, y)\right)-\frac{1}{4} d^{2}(x, y),
$$

where $\sigma_{x}^{y}$ is as in (i) and $m=\sigma_{x}^{y}\left(\frac{1}{2}\right)$ is the point halfway between $x$ and $y$.

We say $\mathcal{X}$ is an Hadamard space if $\mathcal{X}$ is complete and the assertions (i) and (ii) above hold for all points $x, y, z \in \mathcal{X}$. Hadamard spaces are uniquely geodesic, i.e., there exists a unique geodesic between any pair of points.

In this paper, we assume that $\mathcal{X}$ is a geodesically complete Hadamard space, meaning that every geodesic in $\mathcal{X}$ is a subarc of a geodesic which is parametrized on the whole real line. Let $\mathbb{E}^{2}$ be the Euclidean space equipped with the metric

$$
d_{\mathbb{E}^{2}}\left(\left(x_{1}, x_{2}\right),\left(y_{1}, y_{2}\right)\right)=\left(\left(x_{1}-y_{1}\right)^{2}+\left(x_{2}-y_{2}\right)^{2}\right)^{1 / 2} .
$$


A geodesic triangle $\triangle(x, y, z)$ in $\mathcal{X}$ is the union of three points $x, y, z \in \mathcal{X}$ and the geodesic segments joining them. The comparison triangle for $\triangle(x, y, z)$, is a triangle $\Delta(\bar{x}, \bar{y}, \bar{z})$ in $\mathbb{E}^{2}$ such that $d(x, y)=d_{\mathbb{E}^{2}}(\bar{x}, \bar{y}), d(x, z)=d_{\mathbb{E}^{2}}(\bar{x}, \bar{z})$ and $d(z, y)=d_{\mathbb{E}^{2}}(\bar{z}, \bar{y})$. According to this notation: if $a$ is a point on the geodesic segment joining $x, y$, then $\bar{a}$ is its comparison point provided that $d(x, a)=d_{\mathbb{E}^{2}}(\bar{x}, \bar{a})$. Also, the comparison angle $L_{\bar{x}}(\bar{y}, \bar{z})$ is the interior angle of the comparison triangle $\triangle(\bar{x}, \bar{y}, \bar{z})$ at $\bar{x}$.

The first step in defining a subdifferential for a function defined on an Hadamard space $\mathcal{X}$ is to introduce a dual space $\mathcal{X}^{*}$ for $\mathcal{X}$. We denote by $\mathcal{X}^{*}$ the set of all unit speed geodesics of $\mathcal{X}$, i.e., $\mathcal{X}^{*}=\bigsqcup_{x \in \mathcal{X}} \mathcal{X}_{x}^{*}$ where $\mathcal{X}_{x}^{*}$ is the set of all unit speed geodesics of $\mathcal{X}$ starting at $x$. Consider the map $\langle\cdot, \cdot\rangle: \mathcal{X}_{x}^{*} \times \mathcal{X}_{x}^{*} \rightarrow \mathbb{R}$ defined by

$$
\left\langle\gamma_{x}^{y}, \gamma_{x}^{z}\right\rangle=\frac{1}{2}\left[d^{2}(x, z)+d^{2}(x, y)-d^{2}(y, z)\right] .
$$

It is clear that $\left(\left\langle\gamma_{x}^{y}, \gamma_{x}^{y}\right\rangle\right)^{1 / 2}=d(x, y)$; see [Berg and Nikolaev 2008] for more details. Let $\gamma_{x}^{y} \in X_{x}^{*}, \sigma_{z}^{w} \in X_{z}^{*}$ and $D:=\operatorname{dom}\left(\sigma_{z}^{w}\right)=\operatorname{dom}\left(\gamma_{x}^{y}\right)$. Then we say that $\gamma_{x}^{y}$ is parallel to $\sigma_{z}^{w}$ if there exists $C \in \mathbb{R}$ with $d\left(\sigma_{z}^{w}(t), \gamma_{x}^{y}(t)\right)=C$ for all $t \in D$.

\section{The subdifferential of a convex function}

In this section, we present a new definition of the subdifferential of a convex function on an Hadamard space. Note that the function $f: \mathcal{X} \rightarrow \mathbb{R}$ is called convex if, for any geodesic $\gamma$, the composition $f \circ \gamma$ is convex (in the usual sense). Let us start with the definition of the directional derivative for functions on geodesically complete Hadamard spaces.

Definition 2.1. Let $f: \mathcal{X} \rightarrow \mathbb{R}$ be a real-valued function. The directional derivative $D f\left(x ; \gamma_{x}^{z}\right)$ of $f$ at $x \in \mathcal{X}$ in the direction $\gamma_{x}^{z} \in \mathcal{X}_{x}^{*}$ for some $z \in \mathcal{X}$ is defined as

$$
D f\left(x ; \gamma_{x}^{z}\right):=\lim _{t \downarrow 0} \frac{f\left(\gamma_{x}^{z}(t)\right)-f(x)}{t} .
$$

We will use the following remark in the proof of Theorem 2.4.

Remark 2.2. In the case $\mathcal{X}=\mathbb{R}$, the directional derivative of $f$ at $x$ in the direction of $\gamma_{x}^{x+b}$ is defined by

$$
D f\left(x ; \gamma_{x}^{x+b}\right)=\lim _{t \downarrow 0} \frac{f(x+t)-f(x)}{t}
$$

for every $b \in(x, \infty)$. This is the same as the usual directional derivative of $f$ at $x$ in the direction 1 , denoted by $D f(x ; 1)$.

Theorem 2.3. Let $f: \mathcal{X} \rightarrow \mathbb{R}$ be a convex function on $\mathcal{X}$ and consider $\gamma_{x}^{z} \in \mathcal{X}_{x}^{*}$. 
(i) The function $Q: \operatorname{dom}\left(\gamma_{x}^{z}\right) \cap(0, \infty) \rightarrow \mathbb{R}$ defined by

$$
Q(t)=\frac{f\left(\gamma_{x}^{z}(t)\right)-f(x)}{t}
$$

is increasing.

(ii) $D f\left(x ; \gamma_{x}^{z}\right)$ exists and is equal to $\inf _{t} Q(t)$.

(iii) $D f\left(x ; \gamma_{x}^{x}\right)=0$.

Proof. (i) Since $f$ is convex, the function $g(t)=f\left(\gamma_{x}^{z}(t)\right)$, defined on $\operatorname{dom}\left(\gamma_{x}^{z}\right)$, is convex. If $0<t_{1}<t_{2}$, we have

$$
\frac{g\left(t_{1}\right)-g(0)}{t_{1}} \leq \frac{g\left(t_{2}\right)-g(0)}{t_{2}} .
$$

This implies that

$$
\frac{f\left(\gamma_{x}^{z}\left(t_{1}\right)\right)-f(x)}{t_{1}} \leq \frac{f\left(\gamma_{x}^{z}\left(t_{2}\right)\right)-f(x)}{t_{2}},
$$

which means that $Q$ is increasing.

(ii) Assertion (i) implies that for any decreasing sequence of positive numbers $\left\{t_{n}\right\}$ which converges to zero, the sequence $\left\{Q\left(t_{n}\right)\right\}$ is increasing. Hence, $\left\{Q\left(t_{n}\right)\right\}$ has a limit, namely $D f\left(x ; \gamma_{x}^{z}\right)=\inf _{t} Q(t)$.

(iii) For every $x \in \mathcal{X}$ and $t$, we have $\gamma_{x}^{x}(t)=x$. Hence

$$
D f\left(x ; \gamma_{x}^{x}\right)=\lim _{t \downarrow 0} \frac{f\left(\gamma_{x}^{x}(t)\right)-f(x)}{t}=0 .
$$

Theorem 2.4 (mean value theorem). Suppose that $x, y \in \mathcal{X}$, and that $f: \mathcal{X} \longrightarrow \mathbb{R}$ is convex. Then there exists $t_{0} \in(0, d(x, y))$ such that

$$
\frac{f(y)-f(x)}{d(x, y)} \leq D f\left(\gamma_{x}^{y}\left(t_{0}\right) ; \sigma_{\gamma_{x}^{y}\left(t_{0}\right)}^{y}\right) .
$$

Proof. Let $\gamma_{x}^{y}$ be the unit speed geodesic joining $x$ to $y$. Then, $f \circ \gamma_{x}^{y}$ is a real-valued convex function on $[0, d(x, y)]$. By the mean value theorem for convex functions from $\mathbb{R}$ to $\mathbb{R}$, there exist $t_{0} \in(0, d(x, y))$ and $z \in \partial f \circ \gamma_{x}^{y}\left(t_{0}\right)$ such that

$$
\frac{f \circ \gamma_{x}^{y}(d(x, y))-f \circ \gamma_{x}^{y}(0)}{d(x, y)}=z,
$$

where $\partial f \circ \gamma_{x}^{y}\left(t_{0}\right)$ denotes the subdifferential of the real-valued function $f \circ \gamma_{x}^{y}$ at $t_{0}$. We set $w=\gamma_{x}^{y}\left(t_{0}\right)$. For the unit speed geodesic $\sigma_{w}^{y}$,

$$
D f\left(w ; \sigma_{w}^{y}\right)=\lim _{t \downarrow 0} \frac{f \circ \sigma_{w}^{y}(t)-f \circ \sigma_{w}^{y}(0)}{t}=D f \circ \sigma_{w}^{y}(0 ; 1) .
$$

Since the geodesic connecting $w$ and $y$ is unique, we have $\sigma_{w}^{y}(t)=\gamma_{x}^{y}\left(t_{0}+t\right)$ for 
every $t \in[0, d(w, y)]$. Hence, $D f \circ \sigma_{w}^{y}(0 ; 1)=D f \circ \gamma_{x}^{y}\left(t_{0} ; 1\right)$ and $z \leq D f \circ \gamma_{x}^{y}\left(t_{0} ; 1\right)$. Therefore,

$$
\frac{f(y)-f(x)}{d(x, y)} \leq D f\left(\gamma_{x}^{y}\left(t_{0}\right) ; \sigma_{\gamma_{x}^{y}\left(t_{0}\right)}^{y}\right) .
$$

Definition 2.5. Let $f: \mathcal{X} \rightarrow \mathbb{R}$ be a convex function. A geodesic $\gamma_{x}^{z} \in \mathcal{X}_{x}^{*}$ is called the subgradient of $f$ at $x$ if

$$
f(y) \geq f(x)+\left\langle\gamma_{x}^{z}, \sigma_{x}^{y}\right\rangle, \quad \forall y \in \mathcal{X}, \quad \forall \sigma_{x}^{y} \in \mathcal{X}_{x}^{*} .
$$

The set-valued map $\partial f: \mathcal{X} \rightarrow \mathcal{X}^{*}$ is called the subdifferential of $f$ and we call $\partial f(x)$ the subdifferential of $f$ at $x$ : it is the set of all subgradients of $f$ at $x$.

It is worth pointing out that $\partial f(x) \subset \mathcal{X}_{x}^{*}$ for every $x \in \mathcal{X}$. A roughly analogous concept of subdifferential is introduced and investigated on the Hilbert ball in [Reich and Shafrir 1990].

Theorem 2.6. Let $f: \mathcal{X} \rightarrow \mathbb{R}$ be a convex function. Then $\gamma_{x}^{x} \in \partial f(x)$ if and only if $x$ is a minimum point of $f$.

Proof. We know that $\left\langle\gamma_{x}^{x}, \sigma_{x}^{y}\right\rangle=0$ for every $x, y \in \mathcal{X}$ and $\sigma_{x}^{y} \in \mathcal{X}_{x}^{*}$. Hence, if $\gamma_{x}^{x} \in \partial f(x)$, then

$$
f(y) \geq f(x)+\left\langle\gamma_{x}^{x}, \sigma_{x}^{y}\right\rangle=f(x), \quad \forall y \in \mathcal{X}, \forall \sigma_{x}^{y} \in \mathcal{X}_{x}^{*},
$$

which means that $x$ is a minimum point of $f$.

Now assume that $x$ is a minimum point of $f$, so $f(y) \geq f(x)$ for every $y \in \mathcal{X}$. Then

$$
f(y) \geq f(x)+\left\langle\gamma_{x}^{x}, \sigma_{x}^{y}\right\rangle=f(x), \quad \forall y \in \mathcal{X}, \forall \sigma_{x}^{y} \in \mathcal{X}_{x}^{*},
$$

and the proof is complete.

Theorem 2.7. Let $f: \mathcal{X} \rightarrow \mathbb{R}$ be a convex function. If $D f\left(x ; \sigma_{x}^{y}\right) \geq\left\langle\gamma_{x}^{z}, \sigma_{x}^{y}\right\rangle$ for all $y \in \mathcal{X}$ and $\sigma_{x}^{y} \in \mathcal{X}_{x}^{*}$, then $\gamma_{x}^{z} \in \partial f(x)$.

Proof. The relations $D f\left(x ; \sigma_{x}^{y}\right) \geq\left\langle\gamma_{x}^{z}, \sigma_{x}^{y}\right\rangle$ and

$$
f(y)-f(x) \geq \frac{f\left(\sigma_{x}^{y}(s)\right)-f(x)}{s} \geq D f\left(x ; \sigma_{x}^{y}\right)
$$

imply $f(y)-f(x) \geq\left\langle\gamma_{x}^{z}, \sigma_{x}^{y}\right\rangle$, and hence $\gamma_{x}^{z} \in \partial f(x)$.

Corollary 2.8. Let $f: \mathcal{X} \rightarrow \mathbb{R}$ be a convex function. Then $x$ is a minimum point of $f$ if and only if $D f\left(x ; \gamma_{x}^{z}\right) \geq 0$ for each $\gamma_{x}^{z} \in \mathcal{X}_{x}^{*}$.

Proof. If $x$ is a minimum point, then $f\left(\gamma_{x}^{z}(t)\right) \geq f(x)$ for each $z \in \mathcal{X}$ and $t \in \operatorname{dom} \gamma_{x}^{z}$. Hence, $D f\left(x ; \gamma_{x}^{z}\right) \geq 0$. The converse is obvious by Theorem 2.7. 
Lemma 2.9. For each triple of points $x, y, z \in \mathcal{X}$, there exists $w \in \mathcal{X}$ such that $d(x, y)=d(z, w)$ and $\gamma_{x}^{y}$ is parallel to $\sigma_{z}^{w}$.

Proof. Since $\mathcal{X}$ is geodesically complete, there is a unit speed geodesic ray $\gamma_{x}$ connecting $x$ and $y$. By Proposition 9.2.28 in [Burago et al. 2001], there exists a unique unit speed geodesic ray $\sigma_{z}$ starting at $z$, parallel to $\gamma_{x}$. Define $w \in \mathcal{X}$ by $w=\sigma_{z}(d(x, y))$. Then: $d(x, y)=d(w, z)$ and $\gamma_{x}^{y}$ is parallel to $\sigma_{z}^{w}$. Suppose that $\sigma_{z}^{v}$ is another geodesic segment parallel to $\gamma_{x}^{y}$. Since it is also parallel to $\sigma_{z}^{w}$ and $d\left(\sigma_{z}^{w}(0), \sigma_{z}^{v}(0)\right)=0$, we have $d\left(\sigma_{z}^{w}(t), \sigma_{z}^{v}(t)\right)=0$ for each $t \in[0, d(x, y)]$.

We use the notation $\gamma_{x}^{y} \| \gamma_{z}^{w}$ when $\gamma_{x}^{y}$ is parallel to $\gamma_{z}^{w}$ for $x, y, z, w \in \mathcal{X}$. We also denote by $x y$ the line segment between $x, y \in \mathbb{E}^{2}$.

Definition 2.10. (i) The function $P_{x y}: \mathcal{X}_{x}^{*} \longrightarrow \mathcal{X}_{y}^{*}$ defined by $P_{x y}\left(\gamma_{x}^{w}\right)=\gamma_{y}^{v}$ is called the parallel translation of $\gamma_{x}^{w}$ along $\gamma_{x}^{y}$. Here, $v$ is selected such that $d(x, w)=d(y, v)$ and $\gamma_{x}^{w}$ is parallel to $\gamma_{y}^{v}$.

(ii) To define the sum of $\gamma_{x}^{a}$ and $\gamma_{x}^{b}$, we pick a point $c$ such that by $P_{x a}\left(\gamma_{x}^{b}\right)=\gamma_{x}^{c}$ and put $\gamma_{x}^{a}+\gamma_{x}^{b}:=\gamma_{x}^{c}$.

(iii) We define

$$
\begin{aligned}
-\gamma_{x}^{y} & :=P_{y x}\left(\gamma_{y}^{x}\right), \\
\gamma_{x}^{a}-\gamma_{x}^{b} & :=\gamma_{x}^{a}+\left(-\gamma_{x}^{b}\right) .
\end{aligned}
$$

Theorem 2.11. Suppose that $\gamma_{x}^{y}=P_{a x}\left(\gamma_{a}^{b}\right)$ and $\gamma_{x}^{z}=P_{a x}\left(\gamma_{a}^{c}\right)$. Then:

(i) $d(b, c)=d(y, z)$,

(ii) $L_{a}(b, c)=L_{x}(y, z)$,

(iii) $\left\langle\gamma_{a}^{b}, \gamma_{a}^{c}\right\rangle=\left\langle\gamma_{x}^{y}, \gamma_{x}^{z}\right\rangle$,

(iv) $\left\langle-\gamma_{x}^{y}, \gamma_{x}^{z}\right\rangle=\left\langle\gamma_{x}^{y},-\gamma_{x}^{z}\right\rangle$.

Proof. Let $\triangle(\bar{a}, \bar{b}, \bar{c})$ and $\triangle(\bar{x}, \bar{y}, \bar{z})$ be the comparison triangles for $\triangle(a, b, c)$ and $\triangle(x, y, z)$ respectively. By definition, $\left.d\left(\gamma_{a}^{b}(t), \gamma_{x}^{y}(t)\right)=d_{\mathbb{E}^{2}} \overline{\left(\gamma_{a}^{b}(t)\right.}, \overline{\gamma_{x}^{y}(t)}\right)=C$ where $C$ is constant for each $t$. We can assume that $\bar{a} \bar{b} \| \bar{x} \bar{y}$ and $\bar{a} \bar{c} \| \bar{x} \bar{z}$.

This means that $L_{\bar{a}}(\bar{b}, \bar{c})$ and $L_{\bar{x}}(\bar{y}, \bar{z})$ are two angles with parallel sides. They are therefore congruent or supplementary. But since $d_{\mathbb{E}^{2}}\left(\overline{\gamma_{a}^{b}(t)}, \overline{\gamma_{x}^{y}(t)}\right)$ is constant for each $t$, the two angles are congruent.

By a similar argument, we get $L_{\bar{a}}\left(\overline{\gamma_{a}^{b}(t)}, \overline{\gamma_{a}^{c}(t)}\right)=L_{\bar{x}}\left(\overline{\gamma_{x}^{y}(t)}, \overline{\gamma_{x}^{z}(t)}\right)$ for each $t$. Thus, by definition, $L_{a}(b, c)=L_{x}(y, z)$. Moreover, $\triangle(\bar{a}, \bar{b}, \bar{c})$ is congruent to $\triangle(\bar{x}, \bar{y}, \bar{z})$. Then: $d_{\mathbb{E}^{2}}(\bar{b}, \bar{c})=d_{\mathbb{E}^{2}}(\bar{y}, \bar{z})$ and hence $d(b, c)=d(y, z)$. Now by (i) and the definition of $\langle\cdot, \cdot\rangle$, (iii) is obvious.

To prove (iv), suppose that $-\gamma_{x}^{z}=\gamma_{x}^{z^{\prime}}$ and $-\gamma_{x}^{y}=\gamma_{x}^{y^{\prime}}$. Let $\Delta_{1}=\Delta\left(\overline{x_{1}}, \overline{y^{\prime}}, \bar{z}\right)$ and $\triangle_{2}=\Delta\left(\overline{x_{2}}, \bar{y}, \overline{z^{\prime}}\right)$ be the comparison triangles for $\triangle\left(x, y^{\prime}, z\right)$ and $\triangle\left(x, y, z^{\prime}\right)$ respectively. Since $\gamma_{x}^{y^{\prime}} \| \gamma_{y}^{x}$ and $\gamma_{x}^{z^{\prime}} \| \gamma_{z}^{x}$, we can consider $\Delta_{1}$ and $\Delta_{2}$ such that 
$\overline{x_{1}} \overline{y^{\prime}}$ is parallel to $\bar{y} \overline{x_{2}}$ and $\overline{x_{2}} \overline{z^{\prime}}$ is parallel to $\bar{z} \overline{x_{1}}$. Then: $\angle_{\overline{x_{1}}}\left(\bar{z}, \overline{y^{\prime}}\right)=L_{\bar{x}_{2}}\left(\bar{y}, \overline{z^{\prime}}\right)$. Therefore, $\Delta_{1}$ and $\Delta_{2}$ are congruent. Hence, $d_{\mathbb{E}^{2}}\left(\overline{z^{\prime}}, \bar{y}\right)=d_{\mathbb{E}^{2}}\left(\overline{y^{\prime}}, \bar{z}\right)$. It means that $d\left(z^{\prime}, y\right)=d\left(y^{\prime}, z\right)$. Now we have

$$
\begin{aligned}
\left\langle-\gamma_{x}^{y}, \gamma_{x}^{z}\right\rangle & =\left\langle\gamma_{x}^{y^{\prime}}, \gamma_{x}^{z}\right\rangle=\frac{1}{2}\left[d^{2}(x, z)+d^{2}\left(y^{\prime}, x\right)-d^{2}\left(y^{\prime}, z\right)\right] \\
& =\frac{1}{2}\left[d^{2}\left(x, z^{\prime}\right)+d^{2}(x, y)-d^{2}\left(z^{\prime}, y\right)\right]=\left\langle\gamma_{x}^{y}, \gamma_{x}^{z^{\prime}}\right\rangle=\left\langle\gamma_{x}^{y},-\gamma_{x}^{z}\right\rangle .
\end{aligned}
$$

Lemma 2.12. Let $f: \mathcal{X} \rightarrow \mathbb{R}$ be a convex function. Then $\partial f: \mathcal{X} \rightarrow \mathcal{X}^{*}$ is monotone; that is

$$
\left\langle\gamma_{x}^{y}, \sigma_{x}^{z}-P_{y x}\left(\eta_{y}^{n}\right)\right\rangle \leq 0, \quad \forall x, y \in \mathcal{X}, \quad \forall \eta_{y}^{n} \in \partial f(y), \quad \forall \sigma_{x}^{z} \in \partial f(x) .
$$

Proof. Suppose that $\eta_{y}^{n} \in \partial f(y)$ and $\sigma_{x}^{z} \in \partial f(x)$. Thus $f(y)-f(x) \geq\left\langle\gamma_{x}^{y}, \sigma_{x}^{z}\right\rangle$ and $f(x)-f(y) \geq\left\langle\gamma_{y}^{x}, \eta_{y}^{n}\right\rangle$. Note that

$$
\left\langle\gamma_{y}^{x}, \eta_{y}^{n}\right\rangle=\left\langle P_{y x}\left(\eta_{y}^{n}\right), P_{y x}\left(\gamma_{y}^{x}\right)\right\rangle=\left\langle-P_{y x}\left(\eta_{y}^{n}\right), \gamma_{x}^{y}\right\rangle .
$$

Therefore

$$
\left\langle\gamma_{x}^{y}, \sigma_{x}^{z}-P_{y x}\left(\eta_{y}^{n}\right)\right\rangle \leq 0, \quad \forall x, y \in \mathcal{X}, \quad \forall \eta_{y}^{n} \in \partial f(y), \quad \forall \sigma_{x}^{z} \in \partial f(x) .
$$

Let $S$ be a nonempty closed convex subset of $\mathcal{X}$ and $\pi_{S}: \mathcal{X} \longrightarrow S$ be the nearest point map onto $\mathrm{S}$.

Now we need some lemmas to prove the density theorem for the subdifferential of a convex lower semicontinuous function on $\mathcal{X}$.

Lemma 2.13. Let $f: \mathcal{X} \rightarrow \mathbb{R} \cup\{\infty\}$ be a proper, convex and lower semicontinuous function. Suppose that $\left(e, r_{e}\right) \in(\operatorname{epi}(f))^{c}$ and $X_{0}=\left(x_{0}, f\left(x_{0}\right)\right)=\pi_{\mathrm{epi}(f)}\left(e, r_{e}\right)$ with $f\left(x_{0}\right)-r_{e}=1$. Then: $\partial f\left(x_{0}\right) \neq \varnothing$.

Proof. Set $E=\left(e, r_{e}\right)$. By Proposition 2.4 in [Bridson and Haefliger 1999], for each $A=\left(a, r_{a}\right) \in \operatorname{epi}(f)$ not equal to $X_{0}$, we have $L_{X_{0}}(E, A) \geq \frac{\pi}{2}$. Consequently: $\rho^{2}\left(A, X_{0}\right)+\rho^{2}\left(X_{0}, E\right) \leq \rho^{2}(A, E)$, where $\rho$ is the metric of the space $\mathcal{X} \times \mathbb{R}$ defined by

$$
\rho^{2}\left(\left(x_{1}, r_{1}\right),\left(x_{2}, r_{2}\right)\right)=d^{2}\left(x_{1}, x_{2}\right)+\left(r_{2}-r_{1}\right)^{2} \text {. }
$$

Thus

$$
d^{2}\left(a, x_{0}\right)+d^{2}\left(x_{0}, e\right)+\left(f\left(x_{0}\right)-r_{a}\right)^{2}+\left(f\left(x_{0}\right)-r_{e}\right)^{2} \leq d^{2}(a, e)+\left(r_{e}-r_{a}\right)^{2} .
$$

Therefore, we can easily find

$$
\frac{1}{2}\left[d^{2}\left(a, x_{0}\right)+d^{2}\left(x_{0}, e\right)-d^{2}(a, e)\right] \leq\left(r_{a}-f\left(x_{0}\right)\right)\left(f\left(x_{0}\right)-r_{e}\right) .
$$

Since $f\left(x_{0}\right)-r_{e}=1$, we get

$$
\left\langle\gamma_{x_{0}}^{e}, \gamma_{x_{0}}^{a}\right\rangle \leq r_{a}-f\left(x_{0}\right)
$$


for all $a \in \operatorname{dom} f$. Put $r_{a}=f(a)$. Clearly, the above inequality holds for each $a \notin \operatorname{dom} f$. Hence, $\gamma_{x_{0}}^{e} \in \partial f\left(x_{0}\right)$.

It is worth pointing out that since $r_{a}$ in (2-2) (in the proof of Lemma 2.13) can be selected large enough, we get $f\left(x_{0}\right) \geq r_{e}$.

Remark 2.14. The notation $(1-t) a \oplus t b$ is used for some results on Hilbert balls in [Shafrir 1992], on hyperbolic spaces in [Goebel and Reich 1984; Reich and Shafrir 1990] and on Hadamard spaces in [Dhompongsa and Panyanak 2008] to denote the unique point $a_{t}$ such that $d\left(a, a_{t}\right)=t d(a, b)$ and $d\left(a_{t}, b\right)=(1-t) d(a, b)$. Now, if $\left(x_{0}, y_{0}\right)$ and $\left(x_{1}, y_{1}\right)$ are two points in $\mathcal{X} \times \mathcal{Y}$ and $(x, y)$ is a point on the unique geodesic joining them, $(x, y)$ is the unique point satisfying the equations

$$
\begin{aligned}
& \rho\left(\left(x_{0}, y_{0}\right),(x, y)\right)=t \rho\left(\left(x_{0}, y_{0}\right),\left(x_{1}, y_{1}\right)\right), \\
& \rho\left(\left(x_{1}, y_{1}\right),(x, y)\right)=(1-t) \rho\left(\left(x_{0}, y_{0}\right),\left(x_{1}, y_{1}\right)\right)
\end{aligned}
$$

for some $t \in[0,1]$. The point

$$
\left(\gamma_{x_{0}}^{x_{1}}\left(t d\left(x_{0}, x_{1}\right)\right), \gamma_{y_{0}}^{y_{1}}\left(t d\left(y_{0}, y_{1}\right)\right)\right)=\left((1-t) x_{0} \oplus t x_{1},(1-t) y_{0} \oplus t y_{1}\right)
$$

has the same property. Hence

$$
(1-t)\left(x_{0}, y_{0}\right) \oplus t\left(x_{1}, y_{1}\right)=\left((1-t) x_{0} \oplus t x_{1},(1-t) y_{0} \oplus t y_{1}\right)
$$

for all $t \in[0,1]$.

If $x, y \in \mathcal{X}$, we denote by $\llbracket x, y \rrbracket$ the set $\left\{\gamma_{x}^{y}(t): t \in \operatorname{dom} \gamma_{x}^{y}\right\}$.

Lemma 2.15. Let $f: \mathcal{X} \rightarrow \mathbb{R} \cup\{\infty\}$ be a proper, convex and lower semicontinuous function. Suppose that $\left(y_{0}, r_{0}\right) \in(\operatorname{epi}(f))^{c}$ and $X_{0}=\left(x_{0}, f\left(x_{0}\right)\right)=\pi_{\mathrm{epi}(f)}\left(\left(y_{0}, r_{0}\right)\right)$ and $x_{0} \in \operatorname{int}(\operatorname{dom} f)$, where $\operatorname{dom} f=\{x \in \mathcal{X} \mid f(x)<\infty\}$. Then: $r_{0} \neq f\left(x_{0}\right)$.

Proof. Assume by contradiction that $r_{0}=f\left(x_{0}\right)$. Put $Y_{0}=\left(y_{0}, r_{0}\right)$. Let $r$ be a positive number so that $B\left(x_{0}, r\right) \subseteq \operatorname{dom} f$. There is a $\lambda_{0} \in[0,1]$ such that $\gamma_{x_{0}}^{y_{0}}\left(\lambda d\left(x_{0}, y_{0}\right)\right) \in B\left(x_{0}, r\right)$ for the unit speed geodesic $\gamma_{x_{0}}^{y_{0}}$ and for each $\lambda \in\left[0, \lambda_{0}\right]$. First suppose that there exists $x_{1} \in B\left(x_{0}, r\right) \cap \llbracket x_{0}, y_{0} \rrbracket$ such that $f\left(x_{0}\right)<f\left(x_{1}\right)$. Hence, $x_{1}=\gamma_{x_{0}}^{y_{0}}\left(\lambda_{1} d\left(x_{0}, y_{0}\right)\right)$ for some $\lambda_{1} \in\left(0, \lambda_{0}\right)$. Put $X_{1}=\left(x_{1}, f\left(x_{1}\right)\right) \in \operatorname{epi} f$. Then,

$$
\rho^{2}\left(X_{1}, X_{0}\right)+\rho^{2}\left(X_{0}, Y_{0}\right) \leq \rho^{2}\left(X_{1}, Y_{0}\right) .
$$

Putting $\alpha=\left(f\left(x_{1}\right)-f\left(x_{0}\right)\right)^{2}$, we have

$$
\begin{aligned}
& \rho^{2}\left(X_{0}, X_{1}\right)=d^{2}\left(x_{0}, x_{1}\right)+\alpha=\lambda_{1}^{2} d^{2}\left(x_{0}, y_{0}\right)+\alpha, \\
& \rho^{2}\left(Y_{0}, X_{1}\right)=d^{2}\left(y_{0}, x_{1}\right)+\alpha=\left(1-\lambda_{1}\right)^{2} d^{2}\left(x_{0}, y_{0}\right)+\alpha, \\
& \rho^{2}\left(X_{0}, Y_{0}\right)=d^{2}\left(x_{0}, y_{0}\right) .
\end{aligned}
$$


Hence, by (2-3), (2-4) and (2-5), we have

$$
\lambda_{1}^{2} d^{2}\left(x_{0}, y_{0}\right)+\alpha+d^{2}\left(x_{0}, y_{0}\right) \leq\left(1-\lambda_{1}\right)^{2} d^{2}\left(x_{0}, y_{0}\right)+\alpha .
$$

Thus $\lambda_{1}^{2}+1 \leq\left(1-\lambda_{1}\right)^{2}$, and we get the contradiction $\lambda_{1} \leq 0$. Next, consider the case that $f(x) \leq f\left(x_{0}\right)$ for each $x \in B\left(x_{0}, r\right) \cap \llbracket x_{0}, y_{0} \rrbracket$. Let

$$
Y_{n}=\left(1-\frac{1}{n}\right) X_{0} \oplus \frac{1}{n} Y_{0}=\left(y_{n}, r_{n}\right) .
$$

By Proposition 2.4 in [Bridson and Haefliger 1999], $X_{0}$ is the nearest point of epi $(f)$ to each $Y_{n}$, and $\left\{Y_{n}\right\}$ is a sequence converging to $X_{0}$. If $y_{0} \in B\left(x_{0}, r\right)$, then $f\left(y_{0}\right) \leq f\left(x_{0}\right)$. Thus $\left(y_{0}, r_{0}\right)=\left(y_{0}, f\left(x_{0}\right)\right) \in \operatorname{epi}(f)$, which is a contradiction. Therefore, $y_{0} \in\left(B\left(x_{0}, r\right)\right)^{c}$. By Remark 2.14 we have $r_{n}=f\left(x_{0}\right)$ for every $n$, so a similar argument for each $Y_{n}$ shows that $y_{n} \in\left(B\left(x_{0}, r\right)\right)^{c}$. This means that $\left\{y_{n}\right\}$ is a sequence in $\left(B\left(x_{0}, r\right)\right)^{c}$ converging to $x_{0}$. Thus we get the contradiction $x_{0} \notin B\left(x_{0}, r\right)$.

Lemma 2.16. Let $E^{\prime} \in(\operatorname{epi}(f))^{c}$ and $X_{0}=\left(x_{0}, f\left(x_{0}\right)\right)=\pi_{\mathrm{epi}(f)}\left(E^{\prime}\right)$. Then there exists $E=\left(e, r_{e}\right) \in(\mathrm{epi}(f))^{c}$ such that $f\left(x_{0}\right)-r_{e}=1$ and $X_{0}=\pi_{\mathrm{epi}(f)}(E)$.

Proof. Let $\gamma$ be the geodesic joining $X_{0}$ to $E^{\prime}$. Put $E^{\prime}=\left(e^{\prime}, r_{e^{\prime}}\right)$. First suppose that $f\left(x_{0}\right)-r_{e^{\prime}} \geq 1$. Since $\gamma$ is continuous by the intermediate value theorem, the assertion is obvious.

Next, suppose that $f\left(x_{0}\right)-r_{e^{\prime}}<1$. Put

$$
l=\rho\left(X_{0}, E^{\prime}\right) \quad \text { and } \quad s=\frac{l}{f\left(x_{0}\right)-r_{e^{\prime}}} .
$$

Let $\bar{\gamma}$ be the extension of $\gamma$ to $[0, \infty)$ that is the unit speed geodesic ray emanating from $X_{0}$. Put $E=\bar{\gamma}(s)$. We claim that $E$ is the desired point. If $E=\left(e, r_{e}\right)$, then one has $E^{\prime}=\left(1-\frac{l}{s}\right) X_{0} \oplus \frac{l}{s} E$. By Remark 2.14, $e^{\prime}=\left(1-\frac{l}{s}\right) x_{0} \oplus \frac{l}{s} e$ and $r_{e^{\prime}}=\left(1-\frac{l}{s}\right) f\left(x_{0}\right)+\frac{l}{s} r_{e}$. Hence, $f\left(x_{0}\right)-r_{e^{\prime}}=\frac{l}{s}\left(f\left(x_{0}\right)-r_{e}\right)$. Therefore,

$$
f\left(x_{0}\right)-r_{e}=\frac{s}{l}\left(f\left(x_{0}\right)-r_{e^{\prime}}\right)=s \times \frac{f\left(x_{0}\right)-r_{e^{\prime}}}{l}=1 .
$$

Now we prove that $\pi_{\mathrm{epi}(f)}(E)=X_{0}$. Suppose for a contradiction that $\pi_{\mathrm{epi}(f)}(E)=$ $X^{\prime}$ and $X_{0} \neq X^{\prime}$. Then $\angle_{X_{0}}\left(X^{\prime}, E^{\prime}\right) \geq \frac{\pi}{2}$ and $\angle_{X^{\prime}}\left(X_{0}, E\right) \geq \frac{\pi}{2}$. Then the sum of the angles of $\triangle\left(X^{\prime}, X_{0}, E\right)$ is more than $\pi$, which is a contradiction.

The next theorem is a generalization of the density theorem on geodesically complete Hadamard spaces. For a density theorem on Hilbert spaces see [Clarke et al. 1998].

Theorem 2.17. Suppose that $f$ is a proper, convex and lower semicontinuous function. Then $\operatorname{dom}(\partial f(x))$ is dense in $\operatorname{int}(\operatorname{dom} f)$. 
Proof. Given $x_{0} \in \operatorname{int}(\operatorname{dom} f)$, the point $X_{0}=\left(x_{0}, f\left(x_{0}\right)\right)$ is a boundary point of epi $(f)$. So, there exists a sequence $Y_{n}=\left(y_{n}, r_{n}\right)$ in the complement of epi $(f)$ that converges to $X_{0}$. Since epi $(f)$ is convex and closed in $\mathcal{X} \times \mathbb{R}$ there exists a unique point $X_{n}=\left(x_{n}, f\left(x_{n}\right)\right) \in \operatorname{epi}(f)$ such that $\pi_{\text {epi }(f)}\left(Y_{n}\right)=X_{n}$ for each $Y_{n}$. Moreover,

$$
\rho\left(X_{n}, X_{0}\right) \leq \rho\left(X_{n}, Y_{n}\right)+\rho\left(Y_{n}, X_{0}\right) \leq 2 \rho\left(Y_{n}, X_{0}\right),
$$

which implies that $X_{n}$ converges to $X_{0}$. Therefore, the sequence $\left\{x_{n}\right\}$ converges to $x_{0}$ and for every neighborhood $U$ of $x_{0}$, there exists $x_{n} \in U$. By Lemma 2.16, one can assume that $f\left(x_{n}\right)-r_{n}=1$, so by Lemma 2.13, $\partial f\left(x_{n}\right) \neq \varnothing$.

\section{References}

[Ahmadi Kakavandi and Amini 2010] B. Ahmadi Kakavandi and M. Amini, "Duality and subdifferential for convex functions on complete CAT(0) metric spaces", Nonlinear Anal. 73:10 (2010), 3450-3455. MR 2012b:47131 Zbl 1200.53045

[Berg and Nikolaev 2008] I. D. Berg and I. G. Nikolaev, "Quasilinearization and curvature of Aleksandrov spaces”, Geom. Dedicata 133 (2008), 195-218. MR 2008m:53167 Zbl 1144.53045

[Bridson and Haefliger 1999] M. R. Bridson and A. Haefliger, Metric spaces of non-positive curvature, Grundlehren der Mathematischen Wissenschaften 319, Springer, Berlin, 1999. MR 2000k:53038 Zbl 0988.53001

[Burago et al. 2001] D. Burago, Y. Burago, and S. Ivanov, A course in metric geometry, Graduate Studies in Mathematics 33, Amer. Math. Soc., Providence, RI, 2001. MR 2002e:53053 Zbl 0981.51016

[Clarke et al. 1998] F. H. Clarke, Y. S. Ledyaev, R. J. Stern, and P. R. Wolenski, Nonsmooth analysis and control theory, Graduate Texts in Mathematics 178, Springer, New York, 1998. MR 99a:49001 Zbl 1047.49500

[Dhompongsa and Panyanak 2008] S. Dhompongsa and B. Panyanak, "On $\Delta$-convergence theorems in CAT(0) spaces”, Comput. Math. Appl. 56:10 (2008), 2572-2579. MR 2009k:47161 Zbl 1165.65351

[Goebel and Reich 1984] K. Goebel and S. Reich, Uniform convexity, hyperbolic geometry, and nonexpansive mappings, Monographs and Textbooks in Pure and Applied Mathematics 83, Marcel Dekker, New York, 1984. MR 86d:58012 Zbl 0537.46001

[Hosseini and Pouryayevali 2011] S. Hosseini and M. R. Pouryayevali, "Generalized gradients and characterization of epi-Lipschitz sets in Riemannian manifolds", Nonlinear Anal. 74:12 (2011), 3884-3895. MR 2012g:49034 Zbl 1225.49046

[Hosseini and Pouryayevali 2013a] S. Hosseini and M. R. Pouryayevali, "Euler characteristic of epi-Lipschitz subsets of Riemannian manifolds", J. Convex Anal. 20:1 (2013), 67-91. MR 3086441 Zbl 1261.49002

[Hosseini and Pouryayevali 2013b] S. Hosseini and M. R. Pouryayevali, "Nonsmooth optimization techniques on Riemannian manifolds", J. Optim. Theory Appl. 158:2 (2013), 328-342. MR 3084379 Zbl 1273.90110

[Hosseini and Pouryayevali 2013c] S. Hosseini and M. R. Pouryayevali, "On the metric projection onto prox-regular subsets of Riemannian manifolds", Proc. Amer. Math. Soc. 141:1 (2013), 233-244. MR 2988725 Zbl 1277.58005

[Kopecká and Reich 2010] E. Kopecká and S. Reich, "A mean ergodic theorem for nonlinear semigroups on the Hilbert ball", J. Nonlinear Convex Anal. 11:2 (2010), 185-197. MR 2011j:47178 Zbl 1209.47028 
[Reich and Shafrir 1990] S. Reich and I. Shafrir, "Nonexpansive iterations in hyperbolic spaces", Nonlinear Anal. 15:6 (1990), 537-558. MR 91k:47135 Zbl 0728.47043

[Shafrir 1992] I. Shafrir, "Coaccretive operators and firmly nonexpansive mappings in the Hilbert ball”, Nonlinear Anal. 18:7 (1992), 637-648. MR 93d:47100 Zbl 0752.47018

Received April 30, 2014. Revised January 19, 2015.

Mina Movahedi

DEPARTMENT OF MATHEMATICS

FACULTY OF SCIENCES

ALZAHRA UNIVERSITY

TEHRAN

IRAN

m.movahedi18@yahoo.com

m.movahedi@alzahra.ac.ir

DARYOUSH BEHMARDI

DEPARTMENT OF MATHEMATICS

FACULTY OF SCIENCES

ALZAHRA UNIVERSITY

TEHRAN

IRAN

d2beh@yahoo.com

behmardi@alzahra.ac.ir

SEYEDEHSOMAYEH Hosseini

SEMINAR OF APPLIED MATHEMATICS

EIDGENÖSSISCHE TECHNISCHE HoCHSCHULE

CH-8092 ZÜRICH

SWITZERLAND

seyedehsomayeh.hosseini@math.ethz.ch 


\title{
PACIFIC JOURNAL OF MATHEMATICS
}

\author{
msp.org/pjm
}

Founded in 1951 by E. F. Beckenbach (1906-1982) and F. Wolf (1904-1989)

\section{EDITORS}

Don Blasius (Managing Editor)

Department of Mathematics

University of California

Los Angeles, CA 90095-1555

blasius@math.ucla.edu

\author{
Paul Balmer \\ Department of Mathematics \\ University of California \\ Los Angeles, CA 90095-1555 \\ balmer@math.ucla.edu \\ Robert Finn \\ Department of Mathematics \\ Stanford University \\ Stanford, CA 94305-2125 \\ finn@math.stanford.edu \\ Sorin Popa \\ Department of Mathematics \\ University of California \\ Los Angeles, CA 90095-1555 \\ popa@math.ucla.edu
}

\author{
Vyjayanthi Chari \\ Department of Mathematics \\ University of California \\ Riverside, CA 92521-0135 \\ chari@math.ucr.edu \\ Kefeng Liu \\ Department of Mathematics \\ University of California \\ Los Angeles, CA 90095-1555 \\ liu@math.ucla.edu \\ Jie Qing \\ Department of Mathematics \\ University of California \\ Santa Cruz, CA 95064 \\ qing@ cats.ucsc.edu
}

\section{PRODUCTION}

Silvio Levy, Scientific Editor, production@msp.org

\section{SUPPORTING INSTITUTIONS}

ACADEMIA SINICA, TAIPEI

CALIFORNIA INST. OF TECHNOLOGY

INST. DE MATEMÁTICA PURA E APLICADA

KEIO UNIVERSITY

MATH. SCIENCES RESEARCH INSTITUTE

NEW MEXICO STATE UNIV.

OREGON STATE UNIV.

\author{
STANFORD UNIVERSITY \\ UNIV. OF BRITISH COLUMBIA \\ UNIV. OF CALIFORNIA, BERKELEY \\ UNIV. OF CALIFORNIA, DAVIS \\ UNIV. OF CALIFORNIA, LOS ANGELES \\ UNIV. OF CALIFORNIA, RIVERSIDE \\ UNIV. OF CALIFORNIA, SAN DIEGO \\ UNIV. OF CALIF., SANTA BARBARA
}

\author{
Daryl Cooper \\ Department of Mathematics \\ University of California \\ Santa Barbara, CA 93106-3080 \\ cooper@math.ucsb.edu \\ Jiang-Hua Lu \\ Department of Mathematics \\ The University of Hong Kong \\ Pokfulam Rd., Hong Kong \\ jhlu@maths.hku.hk \\ Paul Yang \\ Department of Mathematics \\ Princeton University \\ Princeton NJ 08544-1000 \\ yang@math.princeton.edu
}

These supporting institutions contribute to the cost of publication of this Journal, but they are not owners or publishers and have no responsibility for its contents or policies.

See inside back cover or msp.org/pjm for submission instructions.

The subscription price for 2015 is US \$420/year for the electronic version, and \$570/year for print and electronic.

Subscriptions, requests for back issues and changes of subscribers address should be sent to Pacific Journal of Mathematics, P.O. Box 4163, Berkeley, CA 94704-0163, U.S.A. The Pacific Journal of Mathematics is indexed by Mathematical Reviews, Zentralblatt MATH, PASCAL CNRS Index, Referativnyi Zhurnal, Current Mathematical Publications and Web of Knowledge (Science Citation Index).

The Pacific Journal of Mathematics (ISSN 0030-8730) at the University of California, c/o Department of Mathematics, 798 Evans Hall \#3840, Berkeley, CA 94720-3840, is published twelve times a year. Periodical rate postage paid at Berkeley, CA 94704, and additional mailing offices. POSTMASTER: send address changes to Pacific Journal of Mathematics, P.O. Box 4163, Berkeley, CA 94704-0163.

PJM peer review and production are managed by EditFLOW ${ }^{\circledR}$ from Mathematical Sciences Publishers.

\section{PUBLISHED BY}

\section{mathematical sciences publishers \\ nonprofit scientific publishing}

http://msp.org/

(C) 2015 Mathematical Sciences Publishers 


\section{PACIFIC JOURNAL OF MATHEMATICS}

Volume $276 \quad$ No. $2 \quad$ August 2015

Free evolution on algebras with two states, II

257

MiCHAEL ANSHELEVICH

Systems of parameters and holonomicity of A-hypergeometric systems

281

Christine Berkesch Zamaere, STEPHEN GRIFfeth and EZRA Miller

Complex interpolation and twisted twisted Hilbert spaces

287

FÉlix Cabello SÁnChez, Jesús M. F. Castillo and Nigel J. Kalton

The ramification group filtrations of certain function field extensions

309

JEFFREY A. CASTAÑEDA and QINGQUAN WU

A mean field type flow, II: Existence and convergence

JEAN-BAPTISTE CASTÉRAS

Isometric embedding of negatively curved complete surfaces in Lorentz-Minkowski space

BING-LONG CHEN and LE YIN

The complex Monge-Ampère equation on some compact Hermitian manifolds

JIANCHUN CHU

Topological and physical link theory are distinct

ALEXANDER COWARD and JOEL HASS

The measures of asymmetry for coproducts of convex bodies

Qi GuO, JinFEng GuO and XunLi Su

Regularity and analyticity of solutions in a direction for elliptic equations

YongYANG Jin, DONGSHENG LI and XU-JIA WANG

On the density theorem for the subdifferential of convex functions on Hadamard spaces

Mina Movahedi, Daryoush Behmardi and Seyedehsomayeh

HOSSEINI

$L^{p}$ regularity of weighted Szegó projections on the unit disc

SAMANGi MunASINGHE and Yunus E. ZEYTUnCU

Topology of complete Finsler manifolds admitting convex functions

SORIN V. SABAU and KatSUHIRO SHIOHAMA

Variations of the telescope conjecture and Bousfield lattices for localized categories of spectra

F. LUKE WOLCOTT 